\title{
Quasi-optical approach for inhomogeneous dissipative media with high-order spatial dispersion
}

\author{
A.A. Balakin ${ }^{1}$, E.D. Gospodchikov ${ }^{1,2}$, A.G. Shalashov ${ }^{1,2}$ \\ ${ }^{1}$ Institute of Applied Physics RAS, Nizhny Novgorod, Russia \\ ${ }^{2}$ Budker Institute of Nuclear Physics SB RAS, Novosibirsk, Russia
}

Despite the development of codes for the electromagnetic wave propagation based on a numerical solution of the complete system of Maxwell's equations, asymptotic methods describing wave fields in smoothly inhomogeneous media with complex dielectric response still remain the main instrument of interpretation and optimization of experiments on microwave heating and current drive in magnetic confinement systems, in problems of signal generation in the Earth ionosphere and many other fields of physics and astrophysics. The main and most studied asymptotic approach is based on calculating the geometric-optics ray and absorption along them [1]. However, geometric optics can only qualitatively describe wave propagation and diffraction of the beam in more complex media with strong spatial dispersion and absorption, like, the vicinity of the electron cyclotron resonance in warm plasmas.

The quasi-optical approach is a more accurate asymptotic method, which reduce Maxwell's equations to the evolution equation for the smooth envelope of the wave beam. Such a description allows one to consistently take into account the media anisotropy, spatial inhomogeneity, dispersion, and resonance dissipation within the framework of a unified approach [2]. Its generalization to the case of anisotropic and gyrotropic media with spatial dispersion and dissipation [3] was used to simulate the propagation of quasi-optical wave beams in tokamaks and showed much higher accuracy for resonance absorption in comparison to the previously used approaches [4]. In this paper, we discuss the further generalization of the quasi-optical approach [5] and the features of its application in media of a special kind.

A quasi-optical equation [3] is the equation for the scalar complex amplitude $U$ of the wave beam corresponding to selected electromagnetic mode in smoothly inhomogeneous media

$$
H(\mathbf{r}, \hat{\mathbf{p}})[U(r)]=0 .
$$

Here the momentum operator $\hat{\mathbf{p}}=\nabla / i k_{0}$ is defined as the differentiation operator in the coordinate space normalized on the vacuum wave number $k_{0}=\omega / c$. The simplification of this equation is possible for paraxial wave beams (broad in the wavelength scale) with parameters varying slowly along some curve $\mathbf{r}_{0}$ (the reference ray). One can associate a curvilinear coordinate system $\boldsymbol{r}=(\xi, \tau)$ and canonically conjugate momenta $\hat{\mathbf{p}}=\left(\hat{\mathbf{q}}, \hat{p}_{\tau}\right)$ with the reference ray. Here $\tau$ is the evolutionary coordinate along the reference ray, $\xi$ are two curvilinear coordinates orthogonal to the reference ray, $\hat{\mathbf{q}}=\nabla_{\perp} / i k_{0}$ and $\hat{p}_{\tau}=\partial_{\tau} / i k_{0}$. Assuming that the beam parameters change smoothly along the reference ray, we can represent the scalar amplitude $U$ in the following form

$$
U(r)=\psi(\tau, \xi) e^{i k_{0} \int p_{0}(\tau) d \tau}, \quad|\hat{\mathbf{q}}[\psi]| \ll k_{0} p_{0}|\psi| .
$$

The function $p_{0}(\tau)$ gives the dependence of the "carrier phase" of the wave field along the reference ray. Substituting the field in this form in Eq.(1) and expanding to the first order in powers of $\partial_{\tau} \mathrm{u}$, we obtain the equation for the complex envelope [3]:

$$
\hat{H}[\psi] \approx \hat{H}_{0}[\psi]+\frac{1}{i k_{0}}\left(\frac{\partial \hat{H}_{0}}{\partial p_{0}}\left[\frac{\partial \psi}{\partial \tau}\right]+\frac{1}{2} \frac{\partial^{2} \hat{H}_{0}}{\partial p_{0} \partial \tau}[\psi]\right)=0,
$$

where $\hat{H}_{0}=\hat{H}\left(\mathbf{r}, \hat{\mathbf{q}}+p_{0}(\tau) \boldsymbol{\tau}_{0}\right)$ is the truncated operator with omitted derivatives with respect to $\tau$. The last term gives the pre-exponential factor responsible for the change in intensity due to group deceleration of waves in the geometric optics limit. This term can be eliminated by replacing

$$
\psi=\hat{A}[u], \quad \frac{\partial \hat{H}_{0}}{\partial p_{0}} \frac{\partial \hat{A}}{\partial \tau}+\frac{1}{2} \frac{\partial^{2} \hat{H}_{0}}{\partial p_{0} \partial \tau} \hat{A}=0 .
$$

As a result, we obtain simpler equation

$$
\frac{\partial u}{\partial \tau}=i k_{0} \hat{H}[u], \quad \hat{H}=-\left(\frac{\partial \hat{H}_{0}}{\partial p_{0}} \hat{A}\right)^{-1} \hat{H}_{0} \hat{A} .
$$

This equation describes the evolution of the scalar amplitude of the wave beam $u$ along the $\tau$-axis, taking into account the effects of diffraction, aberrations, spatial dispersion, and dissipation in the paraxial approximation. The physical meaning of the new Hamiltonian $\hat{H}$ is easy to establish if we compare the left and right sides of equation (2). This is the operator of the longitudinal wave number $\hat{p}_{\tau}-p_{0}$, expressed in terms of the transverse derivatives.

Within the approximations made, the quantity

$$
\Pi_{\tau}=\int|u|^{2} d \xi
$$

coincides, with an accuracy up to a dimensional coefficient, with the total energy flux in the wave beam along the reference ray. It is convenient to use the decomposition of the operator $\hat{H}$ into a Hermitian and anti-Hermitian parts

$$
\hat{H}=\hat{H}_{H}+\hat{H}_{A}, \quad \begin{aligned}
& \hat{H}_{H}=\left(\hat{H}+\hat{H}^{+}\right) / 2 \\
& \hat{H}_{H}=\left(\hat{H}-\hat{H}^{+}\right) / 2 i
\end{aligned}
$$

Operators $\hat{H}_{H}, \hat{H}_{A}$ are Hermitian operators in the sense of the standard scalar product $\int a b^{*} d \xi$. As a result, the power deposition can be represented as

$$
\partial_{\tau} \Pi_{\tau}=-2 k_{0} \int u^{*} \hat{H}_{A}[u] d \xi
$$

The main difficulty in the practical use of equation (2) is the uncertainty of the exact form of the op- 
erator $\hat{H}$ for particular physical system. However, any operator can be written in the following form [6]

$$
\hat{H}=\sum_{m, n} \frac{1}{n ! m !}\left(\frac{\partial^{m+n} H}{\partial \xi^{m} \partial \mathbf{q}^{n}}\right) \frac{\xi^{m} \hat{\mathbf{q}}^{n}+\hat{\mathbf{q}}^{n} \xi^{m}}{2} .
$$

Here $H(\tau, \boldsymbol{\xi}, \mathbf{q})$ is some matrix kernel generating the linear operator. For such operator form, the real $H$ always generates the Hermitian operator $\hat{H}$, and the imaginary operator is the anti-Hermitian one. The operator kernel $H=\Sigma H^{(\mathrm{n})}$ represent the sum of corrections to the Hamiltonian in powers of the characteristic inhomogeneity scale $l: H^{(\mathrm{n})} \sim\left(k_{0} l\right)^{-\mathrm{n}}$. In smoothly inhomogeneous media $k_{0} l<<1$, therefore, as a rule, we can confine ourselves to the zero term, that is, the usual Hamiltonian expression obtained in the "locally homogeneous" approximation.

Note, the symmetry of the problem can in a number of cases impose a relation between various corrections in the representation of the operator. For example, a Hamiltonian of the form $H^{(0)}=x^{2} p^{2}$ generates the operator $\hat{H}^{(0)}=-\left(x^{2} \partial_{x x}+2 x \partial_{x}+1\right) / k_{0}^{2}$. However, if it is known that this operator must be proportional only to the field derivatives, then the relation between the zero-order $H^{(0)}$ and the second-order $H^{(2)}$ corrections appears:

$$
\left\{\begin{array}{l}
H^{(0)}=x^{2} p^{2,} \\
H^{(2)}=-1 / k_{0}^{2}
\end{array}\right\} \Rightarrow \hat{H}=-\frac{1}{k_{0}^{2}}\left(x^{2} \partial_{x x}+2 x \partial_{x}\right) .
$$

Lets rewrite representation (5) in terms of the Fourier integral:

$$
\hat{H}[u]=\iint \frac{H(\xi, \mathbf{q})+H(\zeta, \mathbf{q})}{2} e^{i k_{0} \mathbf{q}(\xi-\zeta)} u(\zeta) \frac{d \zeta d \mathbf{q}}{4 \pi^{2} k_{0}^{2}} .
$$

This allows us to write an approximate solution of equation (2) in the form of an operator exponent $[4,5]$ :

$$
\hat{S}[u] \equiv \iint e^{i k_{0} \Delta \frac{H(\xi, \mathbf{q})+H(\zeta, \mathbf{q})}{2}+i k_{0} \mathbf{q}(\xi-\zeta)} u(\tau, \zeta) \frac{d \zeta d \mathbf{q}}{4 \pi^{2} k_{0}^{2}}
$$

Unfortunately, the operator exponent method is justified for media without dissipation. In nonhomogeneous dissipative media with spatial dispersion, the formula (6) can give a solution with exponentially growing energy, even for the case of dissipation within the framework of the geometric optic approximation (ie, for $\operatorname{Im} H>0$ ). The formal reason is that the "dissipative" term $\hat{H}_{A}$ may have negative eigenvalues, since the representation (5) not guarantees the sign-definiteness of eigenvalues.

In fact, this is a known problem of correct media approximation for a physically correct description of the energy absorption of a wave beam [4]. Errors in the approximation of the Hermitian part will only perturb the wave beam phase profile, and their effect will become noticeable on the paths of many diffraction lengths. The systematic errors in the approximation of the anti-Hermitian part directly affect the amplitude of the beam. As a result, an approximate solution can, for example, exponentially increase the beam energy. This problem is especially important for microwave beams of the electron-cyclotron frequency range, when dissipation increases sharply both in the coordinate space and in the space of wave vectors.

The simplest correct approximation guaranteeing the sign-definite nature of the eigenvalues of the antiHermitian part of the operator is to use a model operator that can be represented as the square of some other Hermitian operator. So, we define $\hat{H}_{A}=\hat{G} \hat{G}$, where the new operator is constructed similarly to (6), but with kernel $G(\xi, q) \equiv \sqrt{\operatorname{Im} H(\xi, \mathbf{q})}$.

Thus, using the modified representation for the dissipative part, the quasi-optical equation (2) become

$$
\frac{\partial u}{\partial \tau}=i k_{0} \hat{H}_{H}[u]-k_{0} \hat{G} \hat{G}[u] .
$$

To construct a numerical algorithm for solving this equation, we use the "split-step" method, which consistently takes into account the Hermitian $i k_{0} \hat{H}_{H}[u]$ and anti-Hermitian $k_{0} \hat{G} \hat{G}[u]$ terms

$$
u(\tau+\Delta, \xi) \approx \hat{D} \hat{S}[u] .
$$

Here $\hat{S}$ is defined by the operator exponent (6) with the kernel from the real part of $H$. The operator $\hat{D}$ should correctly describe the weakly damped modes and provide exponential damping at large eigenvalues. One of the simplest operators satisfying the specified conditions is $\hat{D}=1-\hat{T} \hat{T}$ with operator $\hat{T}$ being constructed similarly to (6) for the kernel

$$
T(\xi, \mathbf{q})=\tanh \left(\sqrt{k_{0} \Delta} G(\xi, \mathbf{q})\right)
$$

This gives us the explicit scheme (8) for the quasioptical equation (7), which ensures a stable solution.

The technique developed in this work was used to create quasi-optical code QOOT for numerical simuation of propagation and absorption of quasi-optical microwave beams in a direct magnetic trap. Recently, the code was applied to simulate resonant electroncyclotron plasma heating in a large-scale trap GDT in the Budker Institute [7]. It is shown that under conditions of a large installation with dense hot plasma, the quasi-optical effects of inhomogeneous dissipation can be taken into account in future optimized scenarios of ECR heating.

The work was supported by the Russian Science Foundation (pr. 14-12-01007).

\section{References}

1. Yu. A. Kravtsov and Yu. I. Orlov, Geometrical Optics in Inhomogeneous Media, Springer Ser. on Wave Phenomena (Springer, Berlin, Heidelberg, 1990).

2. A.V. Timofeev, Physics-Uspekhi 48, 609 (2005); G. V. Permitin and A. I. Smirnov, JETP 82, 395 (1996); A.A. Balakin, et.al., JoPD, 40, 4285 (2007).

3. A.A. Balakin, et.al., Plas.Phys.Rep, 33, 302 (2007); A.A. Balakin, Radiophys.Quant.Electron. 55, 472 (2012); A.A. Balakin, Radiophys.Quant.Electron. 55, 502 (2012).

4. A.A. Balakin, et.al., Nucl.Fusion, 48065003 (2008); N. Bertelli, et.al., Nucl.Fusion 50, 115008 (2010).

5. A.G. Shalashov, et.al., Phys.Plasmas 23, 112504

(2016); A.G. Shalashov, et.al., JETP 124, 325 (2017).

6. A.A. Balakin, E.D. Gospodchikov, JoPB, 48, 215701 (2015).

7. P.A. Bagryansky, et.al., PRL, 114, 205001 (2015); P.A.Bagryansky, et.al., Nucl.Fusion, 55, 053009 (2015). 